\title{
Confianza y desconfianza en el sistema público de salud en México
}

FELIPE HEVIA(1)

\section{INTRODUCCIÓN}

La confianza/desconfianza en las sociedades complejas parece ser cada día más un factor clave $^{1-4}$ y el sector salud no escapa a ello ${ }^{5,6}$. Sin embargo, a pesar de su importancia, son pocas las investigaciones que buscan comprender cómo se construye la confianza/desconfianza sobre el sistema público de salud por parte de sus usuarios. Como concluimos en esta investigación, esta pregunta es central puesto que la confianza aparece como un elemento fundamental para tomar decisiones sobre el sistema de salud (público o privado) que se utilizará en caso de existir alguna enfermedad en el hogar.

En la literatura especializada se ha definido a la confianza como un mecanismo de reducción de la complejidad ante un futuro incierto ${ }^{3,4}$. Utilizando la definición de Sztopka, "La confianza es una apuesta acerca de las acciones contingentes futuras de otros (...) Confiar llega a ser una estrategia crucial para tratar con un futuro incierto e incontrolable" 3 . Es decir, frente a la incertidumbre que implica el mundo futuro, la confianza aparece como un mecanismo que nos brinda seguridad y tranquilidad, nos sentimos menos vulnerables. De ahí que resulte en contextos de salud central la confianza: en situaciones de vulnerabilidad (como es padecer una enfermedad) y de incertidumbre (no saber de qué tipo y gravedad de enfermedad se trata) las personas recurren a la confianza como una forma de reducir esta incertidumbre.
Esta investigación se pregunta no sólo por el grado de confianza que tienen los usuarios en el sistema público de salud, sino que intenta identificar los elementos que dotan de contenido dichas representaciones sociales, por medio del análisis de los discursos de los usuarios del Centro de Salud Emiliano Zapata, ubicado en Xalapa. México, un centro de atención primaria de la capital del estado de Veracruz, de 450.000 habitantes ${ }^{7}$.

\section{MATERIAL Y MÉTODO}

Para esta investigación se realizaron 4 grupos focales y 3 entrevistas en profundidad, que se centraron en la calidad de la atención. Fueron transcritos y analizados bajo los parámetros del análisis estructural del discurso ${ }^{8,9}$. Esta información se contrastó con los resultados de la encuesta de Trato Digno desarrollada por la "Cruzada Nacional de la Calidad de los Servicios de Salud" de la Secretaría de Salud y Asistencia del Estado Mexicano ${ }^{10,11}$, en el Centro de Salud Emiliano Zapata, Xalapa. La Cruzada... es una iniciativa del gobierno federal mexicano, que involucra a las secretarías o ministerios del ramo federal y estatales, para introducir la cultura de la calidad en todos los niveles de atención*.

\section{RESULTADOS}

\section{Discursos sociales}

Los discursos sociales pueden ordenarse en

(1) Estudiante de doctorado en Antropología Social del Centro de Investigaciones y Estudios Superiores en Antropología Social (CIESAS-DF) México. heviadelajara@yahoo.com 
Tabla 1. Códigos semánticos significativos en buena atención

\begin{tabular}{|c|c|c|}
\hline & Buena Atención $(+)$ & Mala atención $(-)$ \\
\hline $\begin{array}{c}\text { Capacidades técnicas } \\
\text { del médico }\end{array}$ & Chequeo médico exhaustivo & $\begin{array}{c}\text { No revisan/revisan sólo } \\
\text { por encima }\end{array}$ \\
\hline & $\begin{array}{c}\text { Traspaso adecuado de } \\
\text { información }\end{array}$ & No explican detalles \\
\hline $\begin{array}{c}\text { Calidad cantidad } \\
\text { medicamentos }\end{array}$ & Capacitación & $\begin{array}{c}\text { Recetan medicamentos capacitados } \\
\text { caducos }\end{array}$ \\
\hline & Potencia y dosis adecuada & $\begin{array}{c}\text { No están los medicamentos } \\
\text { en los centros de salud }\end{array}$ \\
\hline Trato humano & $\begin{array}{c}\text { Existencia en los Centros } \\
\text { de Salud }\end{array}$ & $\begin{array}{c}\text { No se ponen en el lugar } \\
\text { de uno } \\
\text { personal de salud } \\
\text { Tiempo dedicado }\end{array}$ \\
& Hay que esperar mucho \\
& Individualidad & $\begin{array}{c}\text { Tratan como enfermedad } \\
\text { y no como personas }\end{array}$ \\
\hline
\end{tabular}

torno a códigos semánticos significativos, los que a su vez implican una valoración del hablante ${ }^{8,9}$. La siguiente tabla resume los códigos o unidades semánticas significativas que refieren los usuarios cuando caracterizan las atenciones médicas.

El primer elemento que utilizan los usuarios para clasificar una atención de buena o mala calidad tiene que ver con las capacidades técnicas del médico. El primer elemento es que exista un chequeo médico exhaustivo por parte del médico:

"A mí no me revisa, no sé, el corazón, la presión, nada. Nomás me pregunta "qué cosa tienes" -tengo dolor en el estómago - "ya, ¿cuáles son tus sintomas del dolor del estómago?" , "pos que tengo diarrea que tengo retortijones" ok. "Bueno pus te voy a recetar esto y esto", pero no me sentó, no me escuchó el estómago, no me pesó, no me tomó la presión ni nada. Y ya, me voy con mi receta y salgo, hasta luego, adiós. Yo creo que la consulta yo he tomado tiempo no dura más de ocho minutos". (Mujer, 35 años) Otro elemento considerado fundamental en los discursos sociales tiene que ver con el traspaso adecuado de información. Pero no basta con "mostrar" hay que "demostrar" también que el médico posee las capacidades técnicas y "comunicativas" para traspasar adecuadamente la información:

"O son, a veces los doctores te pueden dar detalles que uno no sabe... mi mamá lleva tres años utilizando esas cosas para asmáticos (...) y ahora que llegamos, que te digan algo tan elemental como "ah, pos fíjese que los estos tienen cortisona"(...) entonces te apantalla [impresiona] y al apantallarte el doctor, te genera confianza, o sea ah, este sabe", (Mujer, 28 años).

Una variable más en esta categoría tiene que ver con la capacitación que muestra (y demuestra) el médico. Es decir, entre las capacidades técnicas de un médico que da una buena atención, está también que sea uno que siga preparándose:

“ ¿sabes en el caso de los médicos me dices confianza a qué lo ligo en el segundo? Al conocimiento, o sea a todo eso de grados académicos todo eso, yo voy por ejemplo a un consultorio y veo que tiene veinte diplomas de congresos internacionales digo jay si, este sabe! (Hombre, 29 años).

Una segunda categoría que afecta la calidad de la atención tiene que ver con la cantidad y calidad de los medicamentos. Así, los discursos identifican una buena atención con medicamentos que funcionen y que estén disponibles en los establecimientos de salud: -Entrevistador: y me decían que había un problema con los medicamentos, que por eso la atención era regular...

- Si porque no hay medicamentos

\footnotetext{
*Para mayores referencias sobre la Cruzada, ver www.salud.gob.m
} 
- Entrevistador: ¿y qué medicamentos les recetan? - Pus a veces son buenos, pero a veces no (...) [a una señora] le recetaron un medicamento que en vez que ayudarla la perjudicaron (...)y ella está ahora con un médico particular y le dijo que era a causa de los medicamentos que le habían puesto (Mujer, 56 años).

El desabastecimiento de medicamentos en el sistema público de salud en México no es una novedad; sin embargo, en los discursos más se habla de la calidad de los mismos. En particular se hace referencia tanto a la potencia como a las dosis de éstos:

"Si hay medicamentos, pos ya la hice, y si no lo hay, pos regrésate con la doctora pa' decirle que no hay medicamentos y que sustituya ese por otro. Bueno ya, lo sustituye pero sabes que me dice "no hay penicilina, te voy a recetar un sustituto que te va a tardar mucho más en hacerte el efecto porque no es netamente penicilina sino es derivado". (Mujer, 25 años).

Un punto central en la definición de una atención de calidad tiene que ver con el trato que da el personal de salud (médicos y enfermeras) a los pacientes. Cuatro son los principales elementos que construyen una atención personalizada. Aquí se incluye, en primer lugar, el interés y escucha activa que tenga el médico, en segundo, las capacidades empáticas o características de actitud o personalidad de los profesionales, puntos coincidentes con otras investigaciones ${ }^{12-15}$. "[un buen médico] te pone mucha atención, checa cada detalle, escucha cada síntoma que tengas tú que, que platicarle, eh se preocupa por el paciente. (Hombre, 29 años).

Los otros elementos son el tiempo dedicado al paciente (tema inseparables de las capacidades técnicas del profesional) y la individualidad del trato:

"Algunos médicos te atienden porque te tienen que atender, no te hacen caso, vaya, eres como que un número más en la lista ;no? Se cumplen con cubrir su lista de pacientes y anda más. De todas formas perciben un salario con pacientes o sin pacientes". (Mujer, 35 años).

También influye en la percepción de la calidad de la atención la percepción de los tiempos de espera. La idea es que éstos son largos, a pesar de que las mediciones en salas de espera y las encuestas a los usuarios dicen otra cosa. Cabe señalar que la variación de estos tiempos es muy grande, principalmente por el sistema de turnos de atención (se atiende al que llega primero en casos de no estar citados) lo que hace que los primeros, prácticamente, no esperen mientras que los últimos deban esperar por horas.

En sus discursos, los actores diferencian claramente los tiempos de espera dependiendo de la naturaleza de la consulta (morbilidad, cita o urgencia). Aquí vale la pena advertir que un elemento relacionado con este tema es la poca flexibilidad que muestran los facultativos frente a dar preferencia a los citados por sobre los que llegan a morbilidad**, a pesar de la comprensión de los usuarios ante las situaciones urgentes: "Sí, bueno de hecho aquí los mexicanos como que tenemos un poquito de conciencia ¿no?, en que bueno, los que están menos enfermos se esperan "que vamos a atender a la señora que está grave”, esto es lo que tenemos aquí yo eso lo he visto aqui'”. (Mujer, 57 años).

\section{OTRO PUNTO DE VISTA: ENCUESTA DE TRATO DIGNO POR LA CALIDAD DE LOS SERVICIOS DE SALUD}

Para contrastar los discursos, se llevó a cabo la encuesta de trato digno, aplicada a 60 usuarios hombres y mujeres seleccionados al azar ${ }^{10}$. El principal resultado aparece como una paradoja: en general los usuarios perciben recibir una muy buena atención, en sus cuatro elementos constitutivos: En todos los ítems sobre las capacidades técnicas del médico, los porcentajes sobrepasan el 90\% de aprobación (aunque llama la atención que la revisión, por ejemplo, no tenga el $100 \%)$

Tabla 2. Información y explicación por parte del médico

\begin{tabular}{|l|l|r|l|r|l|r|}
\hline & \multicolumn{2}{|c|}{ Si } & \multicolumn{2}{|c|}{ No } & \multicolumn{2}{|c|}{ Nsnc } \\
\hline & $\mathrm{N}^{\mathrm{o}}$ & $\%$ & $\mathrm{~N}^{\mathrm{o}}$ & $\%$ & $\mathrm{~N}^{\mathrm{o}}$ & $\%$ \\
\hline ¿Pudo hablar? & 60 & $100 \%$ & 0 & $0 \%$ & 0 & $0 \%$ \\
\hline ¿Lo examinó? & 57 & $95 \%$ & 3 & $5 \%$ & 0 & $0 \%$ \\
\hline ¿Sabe enfermedad? & 54 & $90 \%$ & 3 & $5 \%$ & 3 & $5 \%$ \\
\hline ¿Explicó Tratamiento? & 56 & $93 \%$ & 3 & $5 \%$ & 1 & $2 \%$ \\
\hline $\begin{array}{l}\text { ¿Sabe qué Medicamentos } \\
\text { tomar? }\end{array}$ & 59 & $98 \%$ & 0 & $0 \%$ & 1 & $2 \%$ \\
\hline ¿Explicó los cuidados? & 57 & $95 \%$ & 2 & $3 \%$ & 1 & $2 \%$ \\
\hline
\end{tabular}


Algo parecido pasa con la cantidad y calidad de medicamentos. Vemos que la satisfacción sobre los medicamentos entregados es alta. A pesar de que muchas recetas no fueron surtidas ni siquiera parcialmente, ${ }^{* * *}$ dos de cada tres usuarios se declararon "satisfechos" o "muy satisfechos".

Tabla 3. Cantidad de Medicamentos

\begin{tabular}{|c|l|c|c|}
\hline & Satisfacción & No. & $\%$ \\
\hline \multirow{2}{*}{\begin{tabular}{c} 
Satisfacción sobre $\begin{array}{c}\text { cantidad de } \\
\text { medicamentos } \\
\text { recibidos }\end{array}$ \\
\cline { 2 - 4 }
\end{tabular}} & $\begin{array}{l}\text { Satisfecho } \\
\text { Sats o menos }\end{array}$ & 40 & $67 \%$ \\
\cline { 2 - 4 } & Insatisfecho & 5 & $18 \%$ \\
\cline { 2 - 4 } & Ns/nc & 4 & $7 \%$ \\
\hline
\end{tabular}

Sobre la calidad de los medicamentos recibidos, hubo un porcentaje alto de respuestas NSNC, esto se debió, probablemente, a dos razones: Era primera consulta en el centro de salud o estaban en control y no recibían "medicamentos". En todo caso, sorprende que para un $10 \%$ de los usuarios entrevistados el medicamento entregado o prescrito no haya solucionado el problema de salud.

Tabla 4. Calidad de Medicamentos

\begin{tabular}{|l|c|c|c|c|c|c|}
\hline & \multicolumn{5}{|c|}{ Sí } & \multicolumn{2}{c|}{ No } & \multicolumn{2}{c|}{ Nsnc } \\
\cline { 2 - 7 } & $\mathrm{N}^{\mathrm{o}}$ & $\%$ & $\mathrm{~N}^{\mathrm{o}}$ & $\%$ & $\mathrm{~N}^{\mathrm{o}}$ & $\%$ \\
\hline $\begin{array}{l}\text { La última vez le } \\
\text { sirvieron los } \\
\text { medicamentos }\end{array}$ & 46 & 77 & 6 & $10 \%$ & 8 & 13 \\
\hline
\end{tabular}

Sobre el trato personalizado, se utilizó el formato que desarrolló la Contraloría General del Estado en sus encuestas y que se aplica en todas las oficinas públicas a nivel estatal. En ella aparece un contundente $100 \%$ de atención cordial

Tabla 5. Calidad del trato

\begin{tabular}{|c|c|r|r|}
\hline \multirow{3}{*}{ Trato médico } & Trato & No. & $\%$ \\
\cline { 2 - 4 } & Cordial & 60 & $100 \%$ \\
\cline { 2 - 4 } & Descortés & 0 & $0 \%$ \\
\hline \multirow{3}{*}{ Trato enfermera } & Prepotente & 0 & $0 \%$ \\
\cline { 2 - 4 } & Cordial & 56 & $93 \%$ \\
\cline { 2 - 4 } & Descortés & 3 & $5 \%$ \\
\cline { 2 - 4 } & Prepotente & 1 & $2 \%$ \\
\hline
\end{tabular}

del médico y un $93 \%$ por parte de la enfermera.

Finalmente, también los tiempos de espera se mantuvieron en rangos aceptables, considerando que la pregunta era sobre la percepción de la espera.*

Tabla 6. Tiempos de espera

\begin{tabular}{|c|lll|}
\hline Pregunta & & $\mathrm{N}^{\mathrm{o}}$ & $\%$ \\
\hline \multirow{3}{*}{$\begin{array}{c}\text { Cuánto tuvo que } \\
\text { esperar }\end{array}$} & $<30$ & 35 & $58 \%$ \\
\cline { 2 - 4 } & $30-60$ & 13 & $22 \%$ \\
\cline { 2 - 4 } & $>60$ & 12 & $20 \%$ \\
\hline \multirow{3}{*}{$\begin{array}{c}\text { Con ese tiempo } \\
\text { quedó usted }\end{array}$} & Satisfecho & 54 & $90 \%$ \\
\cline { 2 - 4 } & Más o menos & 4 & $7 \%$ \\
\cline { 2 - 3 } & insatisfecho & 2 & $3 \%$ \\
\hline
\end{tabular}

En esta encuesta, sin embargo, agregamos una pregunta más no incluida en los formatos oficiales: Por qué razón se atiende en el centro de salud.

\section{DISCUSIÓN}

Si comparamos discursos y encuesta, descubrimos una contradicción: los resultados de las encuestas nos dicen que la atención que se da en el primer nivel es buena, los médicos explican e informan y son cordiales, pero al mismo tiempo, en los discursos y en las conversaciones cotidianas observamos que hay una sensación de atenciones de mala calidad.

Esta aparente contradicción revela dos temas muy importantes. El primero tiene relación con la resolutividad del problema, es decir, la capacidad de solucionar el problema de salud por el que asiste el paciente. Y para eso, debemos responder primero estas preguntas: ¿para qué utilizan los usuarios el sistema público de salud? ¿Es ahí donde realmente resuelven sus principales problemas de salud? En este sentido, la tesis que sostengo es que, finalmente, para los temas de construcción de confianza es la capacidad de resolver el problema de salud, en última instancia la que determina los conceptos de confianza o desconfianza, a pesar de que la atención sea considerada de "buena calidad".

\footnotetext{
**Estas categorías, al igual que las demás, están presentes en los discursos de los usuarios, no son conclusiones mías.

*** Lamentablemente la encuesta aplicada no permite saber cuántos medicamentos le fueron prescritos ni cuantos suministrados.

$* * * *$ En este sentido estos tiempos abarcan desde que el paciente entró al centro hasta que se atendió, y no desde que tenía su hora. Esto permite incluir a los pacientes que asisten por morbilidad aguda y no tienen hora citada.
} 


\section{¿Por qué se atiende en este centro de salud?}

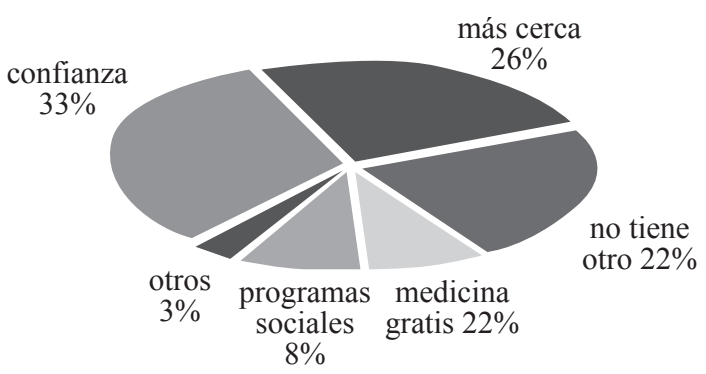

Figura 1. Utilización del servicio de salud

$Y$ en ese sentido es que la gente efectivamente asiste al centro de salud (y responde satisfactoriamente a las encuestas que en él se hacen) pero no soluciona, si puede, sus problemas de salud ahí, prefiere solucionarlos en el sistema privado (con médico particular):

- "Preferimos pagar e ir a un médico particular y sabemos que estamos en buenas manos ¿no?, vamos a un particular si hay dinero. (mujer, 35 años)".

Es decir, los usuarios, para enfermedades más serias o más graves, prefieren asistir con un médico particular. Y en esta decisión influyen directamente los factores que definen una buena atención médica.

En síntesis, las caracterizaciones de confianza (y desconfianza) de los usuarios sobre el SPS se construyen sobre la evaluación que hacen los actores sociales de tres elementos directos y uno indirecto: la capacidad técnica, la calidad de los medicamentos y la atención personalizada y las referencias, familiares, personales y sociales de los sistemas de atención (públicos abiertos, públicos cerrados y privados) donde el riesgo más importante tiene que ver con la resolutividad del sistema. Según estos criterios se caracteriza la relación con el sistema público de salud y constituyen así elementos significativos para la toma de decisiones en salud: dependiendo de la confianza que los usuarios tengan en el sistema abierto de salud pueda resolver su problema, asistirán o no a él.

Pero la confianza como "mecanismo de toma de decisiones" tiene límites. Por un lado, cuando no hay "riesgo" (como en las citas de oportunidades o las vacunaciones) las relaciones no se caracterizan como de confianza o desconfianza y, por otro, la confianza como factor para elegir el sistema de atención en salud está limitada por el dinero, hay un umbral donde se elige el sistema porque no tiene otra posibilidad.

\section{REFERENCIAS}

1. FUKUYAMA F. Confianza Atlántida, Buenos Aires, 1996.

2. GOBIERNO DE CHILE. Confianza social en Chile; desafíos y proyecciones. División de Organizaciones Sociales, Santiago, 2001.

3. SZTOMPKA P. Trust: A sociological Theory.Cambridge. Univ. Press, Cambridge, 1999.

4. MISZTAL B. Trust in modern societies. Polity Press, Londres, 1993.

5. HEVIA DE LA JARA F. Los usuarios ¿confían en lasinstituciones de salud? Centro de Investigaciones y Estudios Superiores en Antropología Social (Tesis Maestría), México, 2003.

6. HEVIA DE LA JARA F. Imágenes de confianza Ponencia presentada en el Segundo Congreso Internacional de Retórica. UNAM, México, 2003.

7. GOBIERNO DE MÉXICO. Instituto Nacional de Estadística Geografía e Informática. Anuario Estadístico de Veracruz CD-ROM INEGI, México, 2003.

8. MARC E. \& PICARD D. La interacción social. Cultura institución y comunicación. Paidós, Barcelona, 1992.

9. MARTINIC S. Análisis estructural: presentación de un método para el estudio de las lógicas culturales CIDE, Santiago, 1992.

10. GOBIERNO DE MÉXICO. Secretaría de salud y asistencia. Programa de mejoría continua de la calidad de la atención médica. Gobierno de México, México, 2000.

11. GOBIERNO DE MÉXICO. Secretaría de salud y asistencia . Cruzada Nacional por la Calidad de los Servicios de Salud. Fundamentos Gobierno de México, México, 2000.

12. LAVADOS M, PAZ R, MOSSÓ L. Evaluación de la relación médico paciente: estudio y reflexión PUCH, Santiago, 1991.

13. HEVIA DE LA JARA F. La Relación Médico Paciente a través de la palabra. Santiago U. de Chile (tesis de grado licenciatura), Santiago, 1999.

14. DOMÍNGUEZ O. La relación médico-paciente: un desafío para la ética moderna CEP, Santiago, 1991.

15. MEEUWESEN L, CAS SHAAP \& VAN DER

STAAK C. Verbal analysis of doctor-patient communication Soc. Sci. Med. 1991. 32(10) pp.1143- 1150 .

Ud. puede comentar éste y otros artículos publicados en la Revista de Salud Pública, enviando un correo electrónico a revistasp@med.uchile.cl 


\title{
La población canina en la Región Metropolitana y sus efectos sobre la salud de las personas
}

\author{
HERNAN VARAS(1)
}

Tradicionalmente el principal problema de salud pública asociado a la población canina es la rabia. En el país existía una condición de enzootia hasta fines de la década de los sesenta, pero la aplicación estratégica y sistemática de las medidas del programa nacional de control logró, a mediados de la década de los ochenta, controlar la presentación de esta enfermedad con casos aislados sin capacidad de generar una epidemia ${ }^{1}$.

Sin embargo, ya en 1997 la población canina en la Región Metropolitana bordeaba el millón de animales, según estudios de la Facultad de Ciencias Veterinarias y Pecuarias de la Universidad de Chile ${ }^{2}$, sólo considerando los canes con dueño, sin contarse en ese momento con una estimación cierta de la cantidad de canes denominados vagabundos o sin dueño.

Más recientemente, de acuerdo a estudios publicados por dicha facultad para el año 2002; realizados con el apoyo de los Departamentos de Higiene y Control Ambiental de las municipalidades de la región aplicando más de 9400 encuestas probabilísticas en 28 de las 34 comunas urbanas y rurales del Gran Santiago; la población canina de la región totalizaba 1.117.192 ejemplares con dueño ${ }^{3 *}$. (Figura 1)

A partir de la cantidad de viviendas habitadas arrojada por el censo de $2002^{4}$, se calculó que la proporción de caninos en residencias ascendía a 3 canes por cada 4 viviendas $(0,76$ canes/vivienda). El promedio de edad de dicha población es levemente mayor a 3 años y medio, por lo que se trata de población canina joven con un alto potencial reproductivo. Con anterioridad Montes (1966) ${ }^{5}$ informó de 280.505 canes para la región, en cambio Matus para $1970^{6}$ encontró una población canina de 361.558 animales es decir un aumento de 20.263 /año.

De acuerdo a las cifras publicadas por Ibarra y cols, el incremento anual entre 1970 y el 2002 es algo superior, llegando a 23613 canes/año (755.634 animales más en ese lapso). De mantenerse esta tendencia; podría esperarse alcanzar en 2010, una población de más de 1.300.000 canes en la Región Metropolitana.

Por otra parte, una proporción importante de la población canina de la región se encuentra circulando por las calles, de acuerdo al estudio de Ibarra, L; Espínola, F; Echeverría, M (2004) ${ }^{7}$ que describe la población estimada del conjunto de "perros callejeros", es decir animales que tienen dueño y una casa y que las personas dejan salir a la calle; "canes supervisados", es decir los animales que circulan junto a su dueño; "perros vagabundos"; que no tienen propietario; y "canes de vecindario", animales que si bien no tienen dueño, los habitantes del lugar, calle o pasaje, mantienen y alimentan sin asumir su propiedad (Figura 2).

Dicha población de circulación libre, fue calculada a partir del promedio de animales estimado por manzana en cada comuna. En base a esta forma de estimación, en las calles de Santiago habría un total de 214.933 canes,

(1) Escuela de Salud Pública. Universidad de Chile. hvaras@med.uchile.cl

*Nivel significación de $99 \%$, error 20\%. Margen no respuesta calc. $20 \%$. No respuesta real: 14,9\% 
correspondiendo en orden de importancia a 112.719 perros callejeros $(52,44 \%) ; 46.443$ perros vagabundos $(21,9 \%)$; que corresponden a la población de más difícil control para los organismos de salud; 37.420 perros supervisados $(16,78 \%)$, y 18.351 perros de vecindario $(8,93 \%$.).En suma, habría 150.139 perros con dueño y 64.794 perros sin dueño. Esta situación ha determinado que se hagan muy relevantes los problemas asociados a la población canina, que se resumen en la Tabla 1.

Entre los problemas descritos destacan los accidentes por mordeduras. De acuerdo a las cifras sobre número de mordeduras, aportadas por la unidad de Zoonosis de la Secretaría Regional Ministerial de Salud ${ }^{8}$ se atendieron durante el trienio 2003-2005, un total de 50.246 mordeduras (Tabla 2), es decir un promedio de 16.749 atenciones/año. en personas que demandaron atención sanitaria. Esta cifra equivaldría a una tasa de 276,3 por 100.000 hbtes. Sin embargo la cifra real de mordeduras es bastante superior ya que de acuerdo a lo publicado por la Facultad de Ciencias Veterinarias y Pecuarias de la Universidad de Chile para la Región ${ }^{9}$, en un estudio poblacional

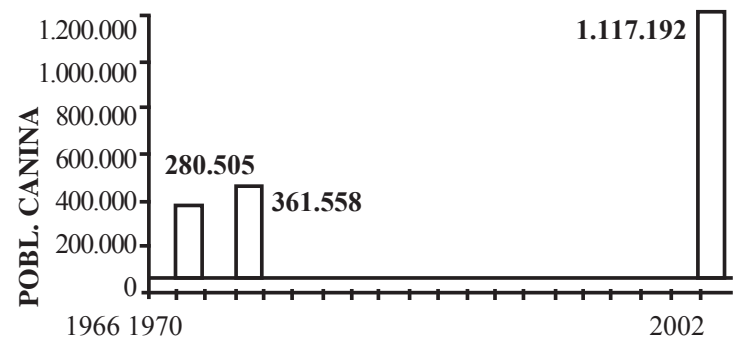

Figura 1. Población canina Región Metropolitana 1966-2002.

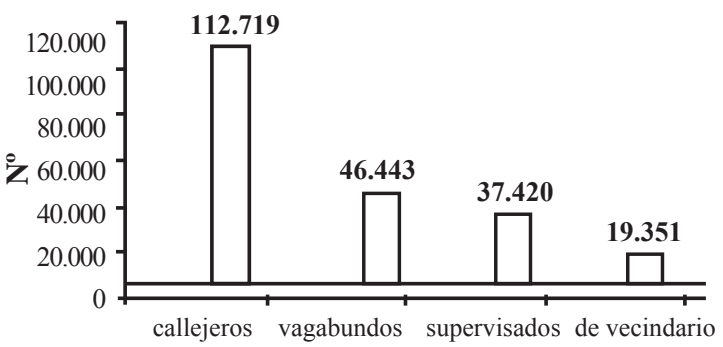

Figura 2. $\mathrm{N}^{\mathrm{o}}$ y tipo de canes de circulación libre en las callles del gran Santiago (2004). se estimó una tasa de mordeduras de 1262 casos por 100.000 hbtes., es decir una cifra 5 veces mayor, lo que implica que se demanda sólo un $22 \%$ de atenciones de salud por esta causa dentro del universo de casos.

Las prestaciones de salud por mordeduras con alguna frecuencia deben incluir el tratamiento de complicaciones. Según Martínez A. ${ }^{10}$; el principal riesgo de las mordeduras es la infección de la herida ya que puede derivar a una artritis séptica, osteomielitis o tendosinovitis.

Esto se traduce en una alta distracción de recursos humanos y financieros necesarios para su resolución. Se estima que el costo unitario de atención de mordeduras, de acuerdo a Urcelay y Aguayo ${ }^{11}$ asciende a US. \$ 45. A un promedio de 16.749 mordeduras/ año, el costo de atención por esta causa se eleva sobre los US \$750.000 anuales.

La población canina, en especial la de circulación libre en las calles de la Región Metropolitana, aparte del problema de las mordeduras a personas, interfiere con su calidad de vida generando inseguridad de desplazamiento en las vías públicas constituyéndose en un peligro para los conductores de vehículos ya que con frecuencia propician accidentes de tránsito,

Por otra parte, contribuyen con frecuencia a la dispersión de basura, la acumulación de fecas en calles y áreas verdes, los malos olores, la proliferación de vectores, la generación de molestias por ruido y la transmisión de enfermedades zoonòticas entre otros importantes problemas de salud pública.

Todo lo expuesto representa un interesante desafío para la autoridad sanitaria regional, toda vez que se requiere coordinar con las autoridades locales y la comunidad una respuesta conjunta al problema de la población canina y sus implicancias en la calidad de vida.

\section{REFERENCIAS}

1. SESMA, CHILE, MINISTERIO DE SALUD. (2001) Problemas asociados a la población canina en la Región Metropolitana. Documento de trabajo. 
2. FACULTAD DE CIENCIAS VETERINARIAS Y PECUARIAS. U DE CH. (1998) Estudio demográfico de la población canina y felina del Gran Santiago.(cit. por Sesma en 1)

3. IBARRA, L; MORALES, M; ACUÑA, P. (2003) Aspectos demográficos de la población de perros y gatos de la ciudad de Santiago de Chile. Avances en Ciencias Veterinarias 18 (1-2) 13-20 ISSN 0706-260X.

4. INSTITUTO NACIONAL DE ESTADÍSTICAS Y CENSO.(INE) (2003) Resultados población y vivienda censo 2002.(citados por Ibarra y cols. en 3 )

5. MONTES, L (1966) Estudio de la población canina en la ciudad de Santiago, su densidad y composición. Tesis: Facultad de Ciencias Pecuarias y Medicina Veterinaria..Universidad de Chile.(73 pp.)

6. MATUS, P; MORALES, M; LOYOLA, R; ROMÁN, D. (1974). Estudio de la población canina del Gran Santiago. Revista de la Sociedad Médico Veterinaria de Chile. Vol. 24 ( pags. 31-42)
7. IBARRA, L; ESPÍNOLA, F; ECHEVERRÍA, M. ( 2004 ).Estimación de la población de perros callejeros y vagabundos en la ciudad de Santiago, Chile (en preparación para publicación)

8. SEREMI SALUD RM (2006) Unidad de Zoonosis. Mordeduras caninas totales anuales en la Región Metropolitana. Trienio 2003-2005. Com. personal.

9. IBARRA, L.; MORALES, M. A.; CÁCERES, L. 2003. Mordeduras a personas por ataque de perros en la ciudad de Santiago, Chile. Avances en Ciencias Veterinarias. Vol 18, (1-2): 41-46.

10. MARTÍNEZ, A. (2005) Microorganismos asociados a infecciones por mordeduras de perros y gatos. Mon. Electr .Patol. Vet. 2 (1) : 1-16).

11. URCELAY, S Y AGUAYO, S (1994) Estimación del costo de mordeduras animales. XII Congreso Panamericano de Ciencias Veterinarias. Acapulco. México. 